\title{
E-cigarette or Vaping Product Use-associated Lung Injury: A Case of an Adult Female Leading to Hospitalization
}

Jeffrey A. Miskoff 1, 2, 3 , Moiuz Chaudhri ${ }^{4}$

1. Medicine, Hackensack Meridian School of Medicine at Seton Hall University, Nutley, USA 2. Medicine - Critical Care Sleep Medicine, Ocean Medical Center, Brick, USA 3. Medicine - Critical Care - Sleep Medicine, Jersey Shore University Medical Center, Neptune City, USA 4. Internal Medicine, Shore Pulmonary, Ocean, USA

Corresponding author: Jeffrey A. Miskoff, jamiskoff@yahoo.com

\begin{abstract}
E-cigarette or vaping is an alternative to traditional cigarette use with potentially devastating consequences. The most recent update from the Centers for Disease Control and Prevention reports a total of 2,561 cases of vaping associated pulmonary injury as of December 27, 2019. This case described a 41year-old female who presented with a clinical picture suggesting of bronchitis; however, a diagnosis of ecigarette or vaping product use-associated lung injury was made.
\end{abstract}

Categories: Family/General Practice, Internal Medicine, Pulmonology

Keywords: vaping, evali, nicotine inhalation, tetrahydrocannabinol, smoking, marijuana

\section{Introduction}

Vaping is the inhalation of vapors created by electronic cigarettes (e-cigarettes) or similar vaping devices. These are battery-powered smoking devices that need cartridges filled with a liquid (flavored or unflavored) containing nicotine and other chemicals [1]. The liquid is heated into a vapor, which is inhaled by the user. Some people use e-cigarettes to inhale marijuana, tetrahydrocannabinol (THC) oil, and other chemicals [2]. Although there are different kinds of vaping e-cigarettes, the most common type is Juul ${ }^{\mathrm{TM}}$ (JUUL Labs, Inc., San Francisco, CA). It looks similar to a universal serial bus (USB) flash drive and can be charged using the USB port [2]. Generally, this type creates less smoke than other e-cigarettes; thus, it can be hard to identify [3]. Dank vape is a counterfeit THC-containing device, and the most commonly reported brand used especially, in the Northeast and Southern United States [3]. The purpose of this case is to increase awareness of e-cigarette or vaping product use-associated lung injury (EVALI) by reporting a case with respiratory, gastrointestinal, and constitutional symptoms combined with abnormal chest imaging. Her treatment included supportive management, systemic steroids, and antibiotics, the typical treatment described in EVALI patients.

\section{Case Presentation}

We present a case of a 41-year-old female who presented to the emergency department (ED) on November 24,2019 , for a cough with brown and green colored sputum and wheezing. Chest X-ray (CXR) was identical to her previous CXR and did not reveal pneumonia-like respiratory processes. The patient was prescribed prednisone and azithromycin, and was later discharged as her acute condition stabilized. The next day, November 25, 2019, the patient presented with shortness of breath, nausea, vomiting, fever, chills, generalized malaise, arthralgia, and inspiratory crackles in the right and left lower lung fields. At this time, pulmonary consultation was requested, and the patient revealed that she was an active smoker, one packyear, and switched to vaping about four to eight weeks before presenting to the ED. According to the patient, she was using THC and nicotine concomitantly for one month. She admitted to using Juul and Blu brand ${ }^{\mathrm{TM}}$ (blu eCigs, Charlotte, NC) for nicotine inhalation but was not sure of the specific brand of THC-containing device she tried in the past. The patient underwent CXR and a chest computed tomography (CT) angiogram, which were remarkable for pulmonary congestion and ground-glass opacities in the left upper lobe and lingula with minimal involvement of the right middle lobe, respectively (Figure 1). 


\section{Cureus}

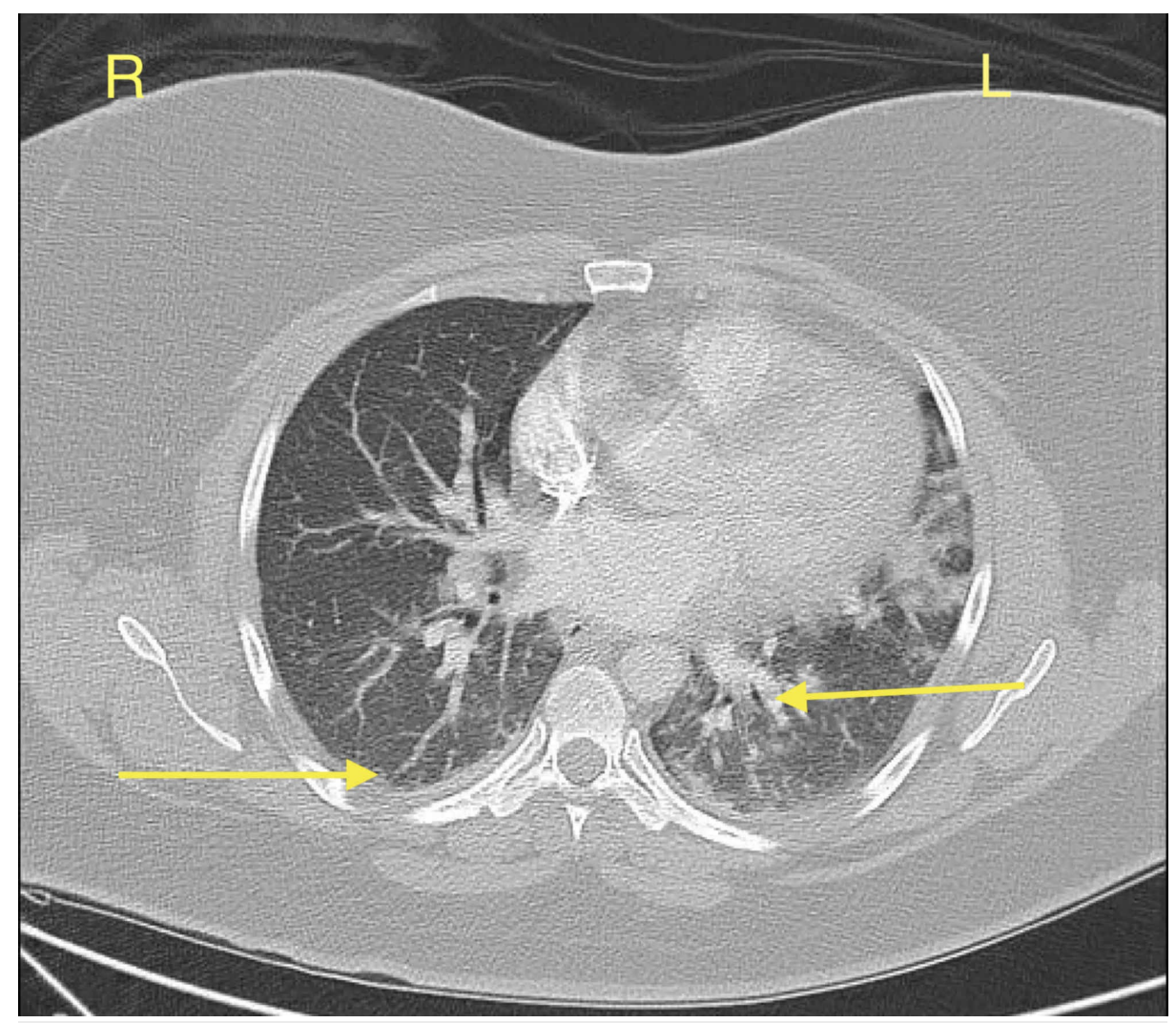

FIGURE 1: Ground-glass opacities in the left upper and right middle lobes.

Physical exam revealed decreased air entry bilaterally and abdominal tenderness localized to the epigastric region. The patient was closely monitored and later discharged on December 2, 2019, with stable respiratory status. The patient was counseled on the recent outbreak of lung injuries and side effects associated with vaping. Furthermore, the patient was encouraged to stop vaping and was encouraged to follow up with a pulmonologist.

\section{Discussion}

EVALI is classified as an acute respiratory illness that can be life-threatening [4]. Vaping can be traced as far back as the 1930s; its addition as an alternative to traditional cigarettes took place in 2003 with the help of a Chinese pharmacist, Hon Lik [5]. In 2007, electronic cigarettes entered the United States marketplace. Initially, it was sold online without specific regulations. Its popularity increased when companies such as Reynolds American and Lorillard made it available in the stores. Data suggest that in 2012, approximately 1.78 million students, grades $6-12$, had tried the product $[6,7]$.

According to the data, more than 2,000 cases of EVALI have been reported to the Centers for Disease Control (CDC) in the last few months, summer 2019 to December 3, 2019 [8,9]. Data from the CDC suggest that the majority of the reported cases include males and below the age of 35 years [4]. Patients diagnosed with EVALI present with symptoms typically seen in acute fibrinous pneumonitis, diffuse alveolar damage, and a mixture of other disease processes such as alveolar hemorrhage, lipoid pneumonia, and acute eosinophilic pneumonia. According to the reports, nicotine, THC, cannabinoid oils are among the most common ingredients behind EVALI $[4,9]$. Analysis suggests that $80 \%$ and $54 \%$ of cases reported to the CDC involved the use of THC and nicotine products, respectively. Furthermore, $40 \%$ of cases involved the use of THC and nicotine concomitantly. In addition, vitamin E acetate, a synthetic form of vitamin E, has been found in bronchoalveolar lavage of patients with EVALI. Majority of the patients present with shortness of breath, cough, chest pain, hemoptysis, and gastrointestinal symptoms with rapid progression to respiratory failure [4].

The patient presented to our care on November 25, 2019, with clinical symptoms suggesting pneumonia, which did not respond to traditional pharmacologic management. Later, a chest CT was remarkable for new ground-glass opacities. Her symptoms in conjunction with recent vaping along with reported EVALI cases suggest this patient might be suffering from vaping-induced respiratory complaints. Her treatment included supportive management, systemic steroids, and antibiotics, typical treatment described in EVALI patients. She improved clinically, and was discharged with an instruction to undergo a pulmonary follow-up as soon 
as possible.

\section{Conclusions}

EVALI epidemic has blown into a full-blown public health crisis forcing CDC to provide regular updates encouraging healthcare providers to be cognizant of clinical presentation. It is important to ask the patient about smoking especially vaping. Lastly, educating patients and their families about this potentially lethal condition despite not being strictly regulated is of the utmost importance.

\section{Additional Information}

\section{Disclosures}

Human subjects: Consent was obtained by all participants in this study. Conflicts of interest: In compliance with the ICMJE uniform disclosure form, all authors declare the following: Payment/services info: All authors have declared that no financial support was received from any organization for the submitted work. Financial relationships: All authors have declared that they have no financial relationships at present or within the previous three years with any organizations that might have an interest in the submitted work. Other relationships: All authors have declared that there are no other relationships or activities that could appear to have influenced the submitted work.

\section{References}

1. Talih S, Salman R, El-Hage R, et al.: Characteristics and toxicant emissions of JUUL electronic cigarettes . Tob Control. 2019, 28:678-680. 10.1136/tobaccocontrol-2018-054616

2. Walley SC, Wilson KM, Winickoff JP, Groner J: A public health crisis: electronic cigarettes, vape, and JUUL . Pediatrics. 2019, 143:e20182741. 10.1542/peds.2018-2741

3. Leavens ELS, Stevens EM, Brett EI, et al.: JUUL electronic cigarette use patterns, other tobacco product use, and reasons for use among ever users: results from a convenience sample. Addict Behav. 2019, 95:178-183. 10.1016/j.addbeh.2019.02.011

4. Layden JE, Ghinai I, Pray I, et al.: Pulmonary illness related to e-cigarette use in Illinois and Wisconsin: preliminary report. N Engl J Med. 2019, Epub ahead of print. 10.1056/NEJMoa1911614

5. Grana R, Benowitz N, Glantz SA: E-cigarettes: a scientific review . Circulation. 2014, 129:1972-1986. 10.1161/CIRCULATIONAHA.114.007667

6. Haardörfer R, Cahn Z, Lewis M, et al.: The advertising strategies of early e-cigarette brand leaders in the United States. Tob Regul Sci. 2017, 3:222-231. 10.18001/TRS.3.2.10

7. Miech R, Patrick ME, O'Malley PM, Johnston LD: What are kids vaping? Results from a national survey of US adolescents. Tob Control. 2017, 26:386-391. 10.1136/tobaccocontrol-2016-053014

8. Chatham-Stephens K, Roguski K, Jang Y, et al.: Characteristics of hospitalized and nonhospitalized patients in a nationwide outbreak of e-cigarette, or vaping, product use-associated lung injury-United States, November 2019. MMWR Morb Mortal Wkly Rep. 2019, 68:1076-1080. 10.15585/mmwr.mm6846e1

9. Schier JG, Meiman JG, Layden J, et al.: Severe pulmonary disease associated with electronic-cigaretteproduct use-interim guidance. MMWR Morb Mortal Wkly Rep. 2019, 68:787-790.

10.15585/mmwr.mm6836e2 